\title{
SCADA IN POWER SYSTEMS
}

\author{
Authors: Matthew N. O. Sadiku, Y. Wang, S. Cui; Sarhan M. Musa \\ ${ }^{1}$ Roy G. Perry College of Engineering \\ Prairie View A\&M University \\ Prairie View, TX 77446 \\ Email: sadiku@ieee.org; yowang@pvamu.edu; sucui@pvamu.edu; smmusa@pvamu.edu
}

\begin{abstract}
Supervisory Control And Data Acquisition (SCADA) is a control system for smooth managing large-scale, automated industrial operations. When applied to electric power industry, it can help the industry to save time and money, reduce operational costs, and improve efficiency. It provides real-time monitoring and automation for smart power grid, a promising power delivery system of the future. This paper provides a brief introduction on the application of SCADA in electric power systems.
\end{abstract}

Key Words: power systems, Supervisory Control And Data Acquisition (SCADA)

\section{INTRODUCTION}

Supervisory control and data acquisition (SCADA) systems control and monitor our critical infrastructures. There are 16 critical infrastructures in US. These include [1]:

energy, waste and water systems, telecommunications, transportation, chemical, dams, emergency services, financial services, commercial facilities, government facilities, critical manufăcturing, defense, food and agriculture, healthcare and public health, and nuclear reactor. These critical infrastructures are interdependent. For example, an attack on the telecommunications sector would affect the energy sector in many ways.

In electric power industry, SCADA systems provide utilities with valuable knowledge and capabilities to deliver power in a reliable and safe manner. A quality SCADA solution is central to effective operation of a utility's most critical and costly distribution, transmission, and generation assets.

Today's SCADA systems, in response to changing business needs, have integrated new communication and network technologies, to make the power grid smart and interactive. They are globally accepted as a means of real-time monitoring and control of electric power systems, particularly for generation, transmission and distribution systems. SCADA systems have been used in electric power systems in the US for more than five decades.

\section{SCADA SYSTEMS}

SCSDA systems consist of software and hardware. The hardware aspect has the following major four components [2,3]:

Sensors: These control relays that directly interface with the managed system. The sensor nodes are used in retrieval of information. They can check specific condition and trigger alarms under anomalous situations.

Remote Terminal Unit (RTU): This is the main component in SCADA system. It is directly connected with sensors, actuators, and meter associated with a control environment. It is basically a real-time programmable logic controllers (PLC) which converts remote station information to digital form for modem to transmit the data. Data acquisition begins with RTUs and includes meter readings and equipment status reports that are communicated to SCADA. RTUs collect I/O data and transfers to remote master unit (MTU) via network interface modules. RTU can use the information to control equipment such as opening or closing a switch. 
Master Terminal Unit (MTU): This is a server communicates with several RTUs by performing reading and writing. It comprises of Local Area Network (LAN) of RCS (Remote Communication Server) and workstations. MTUs provide a human interface to the system and automatically regulate the managed system in response to sensor inputs.

Communications Network: A communication network is needed to transport all the data collected from sensors. This is the glue that holds the automation system together. The network transfers data among servers and the field data interface devices and control units. The system can use different communication technologies such as cable, radio, mobile phones, fibre optics, microwaves, Ethernet or satellites. The communication system connects the supervisory system to the remote terminal units. Communication networks generally support four basic operations: establish communications, terminate communications, write data, and read data. Wireless communication technologies have been incorporated in SCADA systems.

SCADA software package is positioned on top of hardware to which it is interfaced.

They are multi-tasking and are based on a real-time database located in one or more servers. SCADA systems used to run on DOS and UNIX but recently all SCADA vendors have moved to NT and some also to Linux.

A typical SCADA system is shown in Figure 1, while its application in power systems is illustrated in Figure 2. SCADA system architectures have gone through three generations. The first generation employs the wide area network for communication between MTUs. The second generation uses local area networks to link MTUs and RTUs. The third generation uses Internet protocol and wireless technologies [4]. The next generation SCADA systems will move to the cloud, just as other industries have.

\section{ADVANTAGES AND DISADVANTAGES}

The benefits of implementing SCADA systems for power distribution include [5]:

- Increases protection and reliability through automation

- Automation protects workers by enabling problemareas to be detected and addressed automatically

- Eliminates the need for manual data collection

- Alarms and continuous system-wide monitoring enable operators to quickly spot and address problems

- Operators can use powerful trending capabilities to detect future problems provide better routine maintenance of equipment and spot areas for improvement

- Real-time, accurate and consistent information of the system

- Faster fault identification, isolation, and system restoration

- Extensive reporting and statistical data archiving

- Central database and history of all system parameters

The major disadvantages of SCADA systems include [6]:

- Uses proprietary communications and programming

- Lack of compatibility with products from other vendors

- Software is outdated and has known security vulnerabilities

- Any operator with access can shut down the entire system

\section{APPLICATIONS}

SCADA is applied in manufacturing, energy, oil and gas, water and waste, petrochemical, and food production. It is widely used in electric power systems. SCADA solution is central to effective operation of a utility's most critical and costly distribution, transmission, and generation assets. SCADA system is used for remote measurement and control of power system component such as power plant, transmission facilities, and substations. Automation took root in power generating stations earlier than in transmission and distribution.

The main common application of SCADA in power systems include [7]: network connectivity analysis, state estimation, 
load flow application, voltage VAR control, load shed application, fault management and system restoration, loss minimization via feeder reconfiguration, load balancing via feeder reconfiguration, operation monitor, and distribution load forecasting. We consider two of these applications in detail.

Distribution System: Electric power distribution systems involve monitoring and controlling many remote sites, which is difficult. By installing RTUs at substations, SCADA systems have replaced the manual labor to perform electrical distribution tasks and supervise the entire distribution. They provide features like real-time view into the operations and maintain desired voltages, currents and power factors. They perform automatic monitoring, protecting, and controlling of various equipment in distribution systems with the use of RTUs.

Substation Control: Substations are a critical component for maintaining electrical supply and load control. SCADA system continuously monitors the status of various equipment in substation and accordingly sends control signals to them. It performs the operations like bus voltage control, bus load balancing, circulating current control, overload control, etc.

\section{CHALLENGES}

Securing SCADA systems in a power utility environment is challenging due the numerous vulnerabilities inherent in the communications network. As SCADA systems get larger, they are connected to other networks such as the Internet for both economic and technological reasons. The interconnectivity of SCADA systems in the power grid exposes them to a wide range of communication security issues as they become subject a new kinds of threats and cyber attacks such manin-the middle attack, denial-of-service attack, social engineering attack, and insider attack. The attackers' goal is to compromise network security properties such as availability, authentication, confidentiality, or integrity.

The most likely attacking scenario is tapping or hacking the communication line between SCADA server and RTU. To counter this, security devices at two points for encryption and decryption of exchanging information should be installed [8]. Access authorization is also required between the RTUs and master systems. Besides password authorization, other mechanisms for protection include smart cards, firewalls, and intrusion detection systems. Vendors should provide built-in security features in their systems to meet security challenges."

Besides security, installation of SCADA systems is expensive and time-consuming. Updating SCADA software and training employees how to use the new software increases costs. A system may consist of devices from different vendor using different proprietary communications protocols [9].

\section{CONCLUSION}

SCADA systems are basically special computer-based networks and devices which are used in controlling and monitoring infrastructures such as electric power grid. The application of SCADA has simplified the management of the electric power systems worldwide. It will continue to enable power companies to conduct their business in remote and hostile environments.

The SCADA technology is still evolving and we can expect an expansion of the market. The current trend is using transmission control protocol/Internet protocol (TCP/IP)-based systems. More information about SCADA can be found in several books on it available from Amazon.com.

\section{REFERENCES}

[1] L. Goike, "Susceptibility of SCADA systems and the energy sector," Masters Thesis, Utica College, December 2015. [2] "SCADA systems for electrical distribution," https://www.electricaltechnology.org/2015/09/scada-systems-forelectrical-distribution.html

[3] R. B. Roy, "Application of SCADA for controlling electrical power system network," UITS Journal, vol. 1, no. 2, 2012, pp. 85-97.

[4] J. Gao et al., "SCADA communication and security issues," Security and 
Communication Networks, vol. 7, 2014, pp. 175-194.

[5] F. Terezinho, "SCADA systems automate electrical distribution," http://www.indusoft.com/Documentation/WhitePapers/ArtMID/1198/ArticleID/411/SCADA-Systems-Automate-Electrical-Distribution

[6] T. Adams, "SCADA system fundamentals," https://www.cedengineering.com/userfiles/SCADA\%20System\%20Fundamentals.pdf

[7] A. Kumar, "Substation automation using SCADA and financial modeling of 5 MW solar PV," Summer Internship Report,

http://npti.in/Download/MBAInternshipProject/Transmission/22_ashish\%20kumar /Ashish\%20kumar.pdf

[8] D. J. Kang and H. M. Kim, "Development of test-bed and security devices for

SCADA communication in electric power system," Proceedings of the $31^{\text {st }}$ International Telecommunications Energy Conference, October 2009,.

[9] S. Nazir, S. Patel, and D. Patel, "Assessing and augmenting SCADA cyber security: a survey of techniques," Computers \& Security, vol. 70, 2017, pp. 436-454.

\section{AUTHORS}

Matthew N.O. Sadiku is a professor in the Department of Electrical and Computer Engineering at Prairie View A\&M University, Prairie View, Texas. He is the author of several books and papers. His areas of research interest include computational electromagnetics and computer networks. He is a fellow of IEEE.

Yonghui Wang is currently an associate professor with the Department of Engineering Technology, Prairie View A\&M University, Prairie View, TX. His research interests include digital signal processing, image and video coding, and wavelets.

Suxia Cui is an associate professor of Electrical and Computer Engineering Department at Prairie View A\&M University. She has published journal and conference articles in the field of wavelets, image processing, and video coding. Her research interests include data compression, signal classification, image and video processing.

Sarhan M. Musa is a professor in the Department of Engineering Technology at Prairie View A\&M University, Texas. He has been the director of Prairie View Networking Academy, Texas, since 2004. He is an LTD Spring and Boeing Welliver Fellow. 
IJournals: International Journal of Software \& Hardware Research in Engineering

ISSN-2347-4890

Volume 6 Issue 2 February, 2018

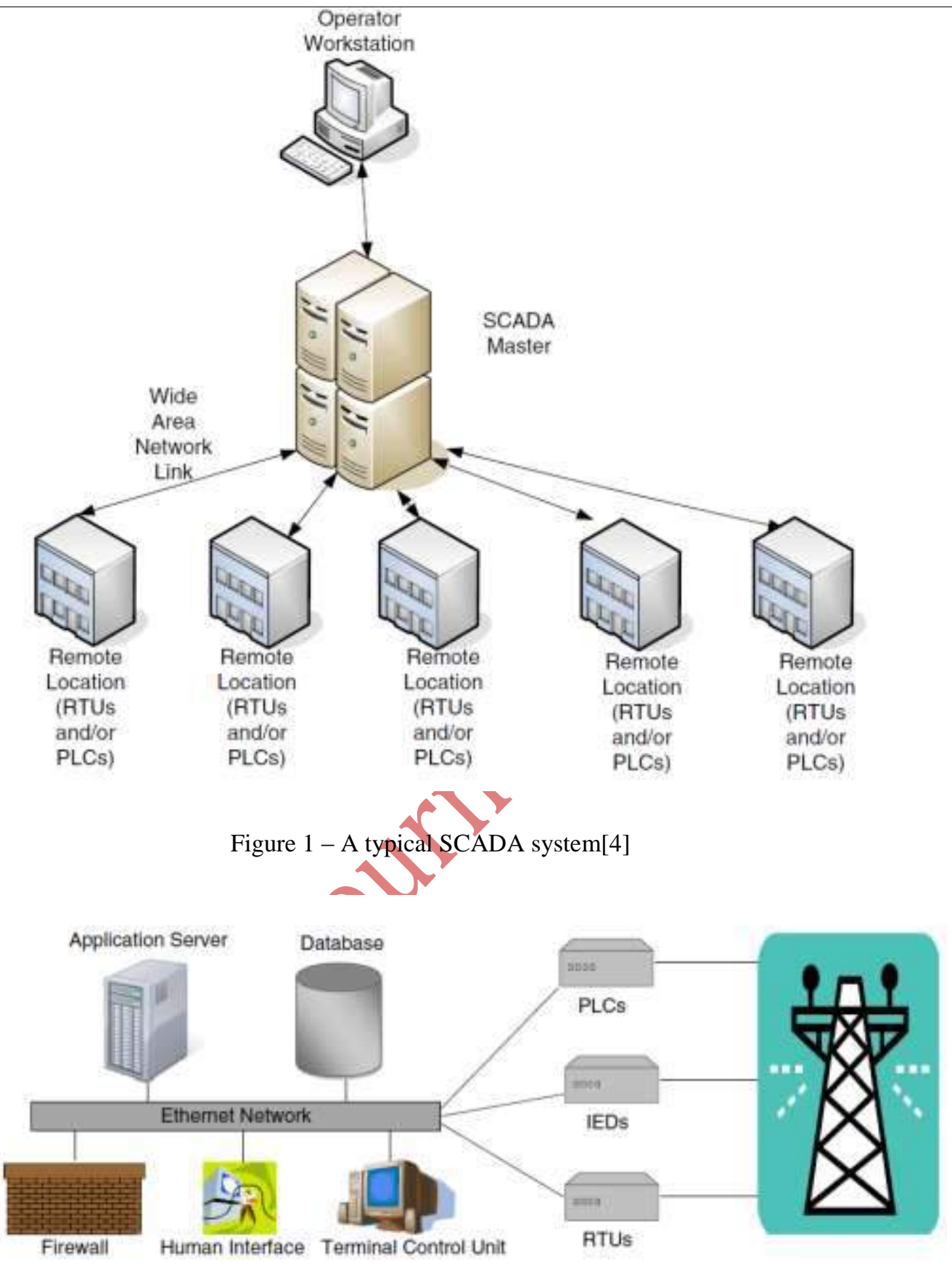

Figure 2-SCADA in power system [4]. 